Теорія Ймовір. та Матем. Статист. Вип. 75, 2006
Theor. Probability and Math. Statist.

No. 75, 2007, Pages 65-69 S 0094-9000(08)00714-X

Article electronically published on January 24, 2008

\title{
ON STOCHASTIC STABILITY OF MARKOV EVOLUTION ASSOCIATED WITH IMPULSE MARKOV DYNAMICAL SYSTEMS
}

UDC 519.21

\author{
V. KOROLYUK AND JE. CARKOVS
}

\begin{abstract}
This paper deals with the family of Cauchy matrices of a linear differential equation dependent on a step Markov process and an impulse type dynamical system rapidly switched by the above process. Applying the stochastic and deterministic averaging procedures according to the invariant measures of the Markov process one achieves a simpler linear differential equation dependent on simpler dynamical systems such as an ordinary differential equation, a differential equation with the right hand side switched by a merger Markov process or a stochastic Itô differential equation. It is proved that under some hypotheses one may successfully apply these resulting evolution families not only to analyzing the initial family on an arbitrary finite time interval but also to describing a time asymptotic of this family.
\end{abstract}

\section{The model, assumptions, and notation}

The mathematical model we analyze consists of:

(SMP) a right continuous Step Markov Process $\left\{y^{\varepsilon}(t), t \geq 0\right\}$ with switching times $\mathbf{S}:=\left\{\tau_{k}^{\varepsilon}, k \in \mathbb{N}\right\}$ given on a discrete metric space $\mathbb{Y}$ by a weak infinitesimal operator [2] $Q^{\varepsilon} v(y):=\frac{1}{\varepsilon} Q_{1} v(y)+Q_{2} v(y)$, where $\varepsilon$ is a small positive parameter, $Q_{j} v(y)=a(y) \sum_{z \in \mathbb{Y}}[v(z)-v(y)] p_{j}(y, z), j=1,2$, and $v(y)$ is an arbitrary bounded measurable mapping $\mathbb{Y} \rightarrow \mathbb{R}$;

(IMDS) an Impulse Markov Dynamical System with rapid switching 9] given as a right continuous $m$-dimensional vector function $\left\{x^{\varepsilon}(t), t \geq 0\right\}$ satisfying

- a differential equation for $t \notin \mathbb{S}$,

- a jump condition for $t \in \mathbb{S}$,

$$
\frac{d x^{\varepsilon}}{d t}=f\left(x^{\varepsilon}(t), y^{\varepsilon}(t), \varepsilon\right)
$$

$$
x^{\varepsilon}(t)=x^{\varepsilon}(t-0)+\varepsilon g\left(x^{\varepsilon}(t-0), y^{\varepsilon}(t-0), \varepsilon\right) ;
$$

(MEF) a Markov Evolution Family or a Markov Multiplicative Cocycle 6, 7] given as a two-parameter family of Cauchy matrices $\left\{X^{\varepsilon}(t, s), t \geq s \geq 0\right\}$ satisfying a linear differential equation in $\mathbb{R}^{n}$ :

$$
\frac{d}{d t} X^{\varepsilon}(t, s)=A\left(x^{\varepsilon}(t), y^{\varepsilon}(t), \varepsilon\right) X^{\varepsilon}(t, s) .
$$

To achieve the limit MEF for (11)-(3) this paper assumes that:

(i) $0<\hat{a}_{1} \leq a(y) \leq \hat{a}_{2}<\infty$ for all $y \in \mathbb{Y}$;

(ii) $\left|p_{2}(y, z)\right| \leq c<\infty, p_{1}(y, z) \geq 0, \sum_{z \in \mathbb{Y}} p_{1}(y, z)=1$ for all $y, z \in \mathbb{Y}$;

2000 Mathematics Subject Classification. Primary 37H10, 34D20. 
(iii) 0 is a simple spectrum point of operator $Q_{1}$ of multiplicity $d$ and

there exists $\rho>0$ such that $\sigma\left(Q_{1}\right) \backslash\{0\} \subset\{z \in \mathbf{C}: \Re z<-\rho\}$;

(iv) $f(x, y, \varepsilon)=f_{1}(x, y)+\varepsilon f_{2}(x, y), g(x, y, \varepsilon)=g_{1}(x, y)+\varepsilon g_{2}(x, y)$, and $f_{j}(x, y)$ and $g_{j}(x, y), j=1,2$, are twice boundedly (on $x$ and $y$ ) continuously differentiable on $x$ functions;

(v) $A(x, y, \varepsilon)=A_{1}(x, y)+\varepsilon A_{2}(x, y)$ and matrices $A_{j}(x, y), j=1,2$, are bounded and continuous on $x$.

To simplify the presentation, the following notation and definitions are used:

- $F_{j}(x, y)=f_{j}(x, y)+a(y) g_{j}(x, y), j=1,2$;

- $\mu_{j}$ are probability measures with disjoint supports $\mathbb{Y}_{j}$ defined as the solutions of the equation $Q_{1}^{*} \mu_{j}=0$, where

$$
\left(Q_{1}^{*} \mu_{j}\right)(y):=\sum_{z \in \mathbb{Y}_{j}} a(z) p_{1}(z, y) \mu_{j}(z)-a(y) \mu_{j}(y), \quad 1 \leq j \leq d ;
$$

- $\mathbf{P}_{0}$ is the projective operator in the kernel of $Q_{1}$ :

$$
\left(\mathbf{P}_{0} v\right)(y):=\sum_{y \in \mathbb{Y}_{j}} \mu_{j}(y) v(y), \quad 1 \leq j \leq d, y \in \mathbb{Y}_{j}
$$

- $\Pi$ is an extension of the potential:

$$
(\Pi v)(y):=\int_{0}^{\infty} \sum_{z \in \mathbb{Y}} P(t, y, z)\left[v(z)-\left(\mathbf{P}_{0} v\right)(z)\right] d t,
$$

where $P(t, y, z)$ is the transition probability of a Markov process 2 corresponding to the infinitesimal operator $Q_{1}$;

- $\lambda_{p}(\varepsilon):=\lim _{t \rightarrow \infty} \sup _{x, y}(p t)^{-1} \ln \mathrm{E}_{x, y}^{s}\left\{\left\|X^{\varepsilon}(t, s)\right\|^{p}\right\}$ is the top Lyapunov $p$-index;

- MEF is said to be asymptotically decreasing with probability one if

$$
\lim _{T \rightarrow \infty} \mathrm{P}\left\{\sup _{t \geq T}\left\|X^{\varepsilon}(t, s)\right\| \geq \delta / x(s)=x, y(s)=y\right\}=0
$$

for any $\delta>0, x \in \mathbb{R}^{n}, y \in \mathbb{Y}$.

\section{LIMIT THEOREMS FOR IMDS}

The problem of asymptotic analysis of dynamical systems with random switching has been discussed in many mathematical and engineering papers. Apparently, A. V. Skorokhod [8] was the first mathematician to have proved that the probabilistic limit theorems may be successfully used for differential equations with right parts dependent on a step Markov process (English edition, [8]). The approach proposed by A. V. Skorokhod and developed by many authors (see, for example, [1] and references there) makes it possible to apply for asymptotic analysis of IMDS (1)-(3) not only the averaging procedure but also the phase merger procedure [5, 10] and diffusion approximation. Some of the above-mentioned results are written out below.

Theorem 1 ([3, 9]). If $\operatorname{dim} \mathbf{P}_{0}=d>1$, then for any $y \in \mathbb{Y}_{k}, k \in\{1, \ldots, d\}, x \in \mathbb{R}^{m}$, $s \geq 0$, and $T>0$ the family of processes $\left\{x^{\varepsilon}(s+t), x^{\varepsilon}(s)=x, t \in[0, T]\right\}$ weakly converges as $\varepsilon \rightarrow 0$ to the solution $\{\hat{x}(t), t \in[0, T]\}$ of the differential equation

$$
\frac{d \hat{x}(t)}{d t}=\hat{F}_{1}(\hat{x}(t), \hat{y}(t))
$$

with initial condition $\hat{x}(0)=x$, where $\hat{F}_{1}\left(x, \mathbb{Y}_{k}\right)=\sum_{z \in \mathbb{Y}_{k}} F_{1}(x, z) \mu_{k}(z)$ and $\hat{y}(t)$ is the merger (or lumping [5]) homogeneous step Markov process that starts from the point 
$\hat{y}(0)=\mathbb{Y}_{k}$ and is given on the merger state space $\hat{\mathbb{Y}}:=\left\{\mathbb{Y}_{j}, j=1, \ldots, d\right\}$ by the infinitesimal matrix $\gamma_{j}^{k}:=\sum_{y \in \mathbb{Y}_{k}} \sum_{z \in \mathbb{Y}_{j}} a(y) p_{2}(y, z) \mu_{k}(y)$ for any $j \neq k$.

Theorem 2 ([1, 9]). If $d=1$, the family of solutions of IMDS,

$$
\left\{x^{\varepsilon}(s+t), x^{\varepsilon}(s)=x, t \in[0, T]\right\},
$$

converges in probability as $\varepsilon \rightarrow 0$ to the solution $\{\bar{x}(t)\}$ of an averaged equation

$$
\frac{d \bar{x}}{d t}=\sum_{y \in \mathbb{Y}} F_{1}(x, y) \mu_{1}(y):=\bar{F}_{1}(\bar{x})
$$

with initial condition $\bar{x}(0)=x$ for any $r>0, s \geq 0$, and $T>0$ uniformly in $y \in \mathbf{Y}$, $x \in\left\{u \in \mathbb{R}^{m}:|u| \leq r\right\}, s \geq 0$, and $t \in[0, T]$,

$$
\lim _{\varepsilon \rightarrow 0} \sup _{y,|x|<r} \mathrm{P}_{x, y}\left(\sup _{0 \leq t \leq T}\left|x^{\varepsilon}(t)-\bar{x}(t, x)\right|>\delta\right)=0, \quad \delta>0, r>0, T>0 .
$$

If $\bar{F}_{1}(x) \equiv 0$, then for any $x \in \mathbb{R}^{m}, y \in \mathbb{Y}$, and $T>0$ the family

$$
\left\{x^{\varepsilon}(s+t / \varepsilon), x^{\varepsilon}(s)=x, t \in[0, T]\right\}
$$

weakly converges as $\varepsilon \rightarrow 0$ to the diffusion Markov process $\{\tilde{x}(t), t \in[0, T], \tilde{x}(0)=0\}$ with weak infinitesimal operator

$$
\mathcal{L}_{0}:=(b(x), \nabla)+(\sigma(x) \nabla, \nabla),
$$

where

$$
b(x)=\sum_{y \in \mathbb{Y}}\left\{F_{2}(x, y)+\left[\Pi D F_{1}(x, y)\right] F_{1}(x, y)\right\} \mu(y)-\sum_{y \in \mathbb{Y}}\left[D F_{1}(x, y)\right] g_{1}(x, y) \mu(y),
$$

$\nabla$ is a vector gradient in $\mathbb{R}^{m}, D$ is a Fréchet derivative, and the positive symmetric matrix $\sigma(x)$ is defined by the equality

$(\sigma(x) z, z)=\sum_{y \in \mathbb{Y}}\left\{\left(F_{1}(x, y), z\right)\left(\Pi F_{1}(x, y), z\right)-\left(g_{1}(x, y), z\right)\left(2 f_{1}(x, y)+a(y) g_{1}(x, y), z\right)\right\} \mu(y)$ with an arbitrary vector $z \in \mathbb{R}^{m}$.

Corollary 1. Under the assumptions of Theorem 2, the normalized deviation family

$$
\left\{\zeta^{\varepsilon}(t), t \in[0, T]\right\}
$$

defined by an equality $\zeta^{\varepsilon}(t):=\left[x^{\varepsilon}(s+t)-\bar{x}(t)\right] / \sqrt{\varepsilon}$ weakly converges as $\varepsilon \rightarrow 0$ to the solution $\zeta^{0}(t)$ of the Itô stochastic equation with drift $D \bar{F}(\bar{x}(t)) \zeta^{0}(t)$ and diffusion matrix $\sigma(\bar{x}(t))$ for any $x \in \mathbb{R}^{m}, y \in \mathbb{Y}, s \geq 0$, and $T>0$.

It should be mentioned that in spite of the fact that the above result has been developed for the analysis of dynamical systems on a finite time interval, one may successfully apply that also to (11)-(2) as $t \rightarrow \infty$ [9].

\section{Asymptotic analysis of MEF}

Lemma 1. Under the above assumptions MEF (31) is exponentially decreasing for any sufficiently small $\varepsilon>0$ with probability one if and only if there exists a positive number $p$ such that the top Lyapunov $p$-index $\lambda_{p}(\varepsilon)$ is negative.

To formulate the asymptotic theorems for MEF (3) we will use a merger MEF defined as the family of Cauchy matrices of the linear differential equation

$$
\frac{d \hat{X}}{d t}=\hat{A}_{1}(\bar{x}(t), \hat{y}(t)) \hat{X}(t)
$$


where $\left.\hat{A}_{1}\left(x, \mathbb{Y}_{k}\right)=\sum_{z \in \mathbb{Y}_{k}} A_{1}(x, z)\right)$, and an average $M E F$ defined as the family of Cauchy matrices of the linear differential equation

$$
\frac{d \bar{X}}{d t}=\bar{A}(\bar{x}(t)) \bar{X}(t)
$$

where $\bar{A}_{1}(x):=\sum_{y \in \mathbb{Y}} A_{1}(x, y) \mu(y)$.

Theorem 3. If the trivial solution of the differential equation (6) is uniformly (with respect to the initial points $\hat{x}(0)=x$ and $\hat{y}(0)=\mathbb{Y}_{j}$ ) almost surely asymptotically stable, then under the assumptions of Theorem 1 the Lyapunov p-index $\lambda_{p}(\varepsilon)$ is negative for any sufficiently small positive numbers $\varepsilon$ and $p$.

Theorem 4. If $d=1$ and the trivial solution of the differential equation (7) is uniformly (with respect to the initial point $\bar{x}(0)=x$ ) asymptotically stable, then under the assumptions of Theorem 2 the Lyapunov $p$-index $\lambda_{p}(\varepsilon)$ is negative for any $p$ and a sufficiently small positive number $\varepsilon$.

These theorems permit us to simplify the time asymptotic analysis of (3) and to deduce a sufficient condition for exponential decreasing. For that one can use, for example, the well-developed second Lyapunov method for deterministic or Markov multiplicative cocycles (see [7] and references there) or a sufficiently simple method for analyzing the mean square stability [3].

Theorem 5. If $d=1, A_{1}(x, y)=0$ and the trivial solution of the differential equation

$$
\frac{d \tilde{X}}{d t}=\bar{A}_{2}(\tilde{x}(t)) \tilde{X}(t)
$$

is uniformly (with respect to the initial point $\tilde{x}(0)=x$ ) almost surely asymptotically stable, then under the assumptions of Theorem 2 the Lyapunov p-index $\lambda_{p}(\varepsilon)$ is negative for any sufficiently small positive numbers $\varepsilon$ and $p$.

Corollary 2. If $d=1$ and the trivial solution of the differential equation

$$
\frac{d \tilde{X}}{d t}=\bar{A}_{1}\left(\bar{x}(t)+\sqrt{\varepsilon} \zeta_{0}(t)\right) \tilde{X}(t)
$$

with $\zeta_{0}(t)$ from Corollary 1 is uniformly (with respect to the initial point $\tilde{x}(0)=x$ ) almost surely asymptotically stable for any sufficiently small positive number $\varepsilon$, then under the assumption of Corollary 1 the Lyapunov p-index $\lambda_{p}(\varepsilon)$ is negative for any sufficiently small positive numbers $\varepsilon$ and $p$.

Using the above results one can take advantage of the covariance method proposed in 4 for the asymptotic analysis of linear differential equations with diffusion coefficients.

\section{BIBLIOGRAPHY}

1. B. V. Anisimov, Random Processes with Discrete Component. Limit Theorems, Kiev Univ., Kiev, 1988. (Russian)

2. E. B. Dynkin, Markov Processes, Springer-Verlag, Berlin, 1965. MR0193671 (33:1887)

3. L. Katafygiotis and Ye. Tsarkov, Mean square stability of linear dynamical systems with small Markov perturbations. I. Bounded coefficients, Random Oper. and Stoch. Equ. 4 (1996), 149170. MR1399076 (98h:60084)

4. L. Katafygiotis and Ye. Tsarkov, Mean square stability of linear dynamical systems with small Markov perturbations. II. Diffusion coefficients, Random Oper. and Stoch. Equ. 4 (1996), 257278. MR 1414878 (98h:60085)

5. V. S. Korolyuk and A. F. Turbin, Limit theorems for Markov random evolutions in the scheme of asymptotic state lumping, Lect. Not. Math. 1021 (1983), 83-88. MR0735999 (86b:60119)

6. V. S. Korolyuk and A. V. Swishchuk, Semi-Markov Random Evolution, Kluwer Academic Publishers, Dordrecht, 1995. MR1472977 (98e:60145) 
7. V. I. Oseledec, A multiplicative ergodic theorem. Lyapunov characteristic numbers for dynamical systems, Transactions of Moscow Mathematical Society 19 (1968), 197-231. MR0240280 $(39: 1629)$

8. A. V. Skorokhod, Asymptotic Methods in the Theory of Stochastic Differential Equations, AMS, Providence, RI, 1989. MR1020057 (90i:60038)

9. Ye. Tsarkov, Asymptotic methods for stability analysis of Markov impulse dynamical systems, Advances of Stability Theory of the End of XXth Century. Stability and Control: Theory, Methods and Applications, Gordon and Breach Science Publishers, London, 2000, pp. 251-264.

10. V. S. Korolyuk, Stability of autonomous dynamical systems with rapid Markov switching, Ukr. Math. J. 43 (1991), 1176-1181. MR1149579 (93i:34103)

Institute of Mathematics, National Academy of Sciences of Ukraine, Tereshchenkivs'ka Street, 3, Kyiv 4, Ukraine

Department of Probability Theory and Mathematical Statistics, Riga Technical UniverSity, Meza Street, 1/4, Riga, Latvia

E-mail address: carkovs@livas.lv

Received 3/NOV/2005

Originally published in English 